\title{
Diacronie
}

Studi di Storia Contemporanea

$N^{\circ} 10,2$ | 2012

Digital History: la storia nell'era dell'accesso

\section{Ce que le numérique fait à l'historien.ne}

Entretien avec Claire Lemercier

Elisa Grandi et Émilien Ruiz

\section{(2) OpenEdition \\ Journals}

\section{Édition électronique}

URL : http://journals.openedition.org/diacronie/2780

DOI : 10.4000/diacronie. 2780

ISSN : 2038-0925

Éditeur

Association culturelle Diacronie

Référence électronique

Elisa Grandi et Émilien Ruiz, « Ce que le numérique fait à l'historien.ne », Diacronie [En ligne], № 10,

2 | 2012, document 1, mis en ligne le 29 juin 2012, consulté le 02 mai 2019. URL : http://

journals.openedition.org/diacronie/2780; DOI : 10.4000/diacronie.2780 


\section{Diacronie}

1/

\section{Ce que le numérique fait à l'historien.ne Entretien avec Claire Lemercier}

par Elisa GRANDI et Émilien RUIZ

Claire Lemercier répond aux questions posées par Elisa Grandi et Émilien Ruiz sur le thème de la Digital History et sur le rôle que l'historien va jouer dans le développement du numérique.

Les questions ont étés élaborées par Elisa Grandi et Émilien Ruiz et l'interview a été réalisée au mois de mai 2012 à Paris.

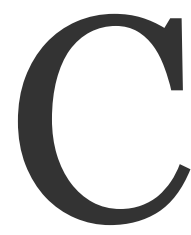

laire Lemercier est chargée de recherche au CNRS, au Centre de sociologie des organisations ${ }^{1}$, après avoir travaillé à l'Institut d'histoire moderne et contemporaine $^{2}$. Surtout spécialiste du XIX ${ }^{e}$ siècle en France, elle a étudié différentes institutions et formes de régulation économiques, dont dernièrement les tribunaux très spéciaux que sont les conseils de prud'hommes et les tribunaux de commerce. Elle vient ainsi de soutenir, le 19 mars 2012, une habilitation à diriger les recherche (HDR) sur un dossier intitulé Sociologie historique des institutions économiques dans la France du $\mathrm{XIX}^{\mathrm{e}}$ siècle, dont le manuscrit inédit est intitulé Un modèle français de jugement des pairs. Les tribunaux de commerce, 1790$1880^{3}$.

Parallèlement, elle a mené différentes activités éditoriales (notamment à Revues.org 4 , Histoire \& Mesure5 et pour la collection "Repères" des éditions La Découverte) et de

${ }^{1}$ Centre de sociologie des organisations (CSO), UMR 7116 CNRS-Sciences po.,

URL : < http://www.cso.edu/home.asp > [consulté le 3 mai 2012].

${ }^{2}$ Institut d'histoire moderne et contemporaine (IHMC), UMR 8066 CNRS-ENS,

URL : < http://www.ihmc.ens.fr/ > [consulté le 3 mai 2012].

3 Lire en ligne sur le serveur TEL (Thèses en ligne),

URL: < http://tel.archives-ouvertes.fr/tel-oo685544 > [consulté le 3 mai 2012].

4 Revues.org. Plateforme de revues et collections de livres en sciences humaines et sociales,

URL : <http://www.revues.org/ > [consulté le 3 mai 2012]. 
vulgarisation des méthodes quantitatives. Parmi ses publications figurent : Un si discret pouvoir. Aux origines de la chambre de commerce de Paris, 1803-1853, Paris, La Découverte, 2003, ouvrage tiré de sa thèse de doctorat en histoire ${ }^{6}$; et (avec Claire Zalc), Méthodes quantitatives pour l'historien, Paris, La Découverte, 2008 - tiré d'un enseignement dispensé depuis 2005 à l'ENS et à l'EHESS : "L'histoire et l'historien.ne face au quantitatif" - assorti d'un site web annexe "Quanti IHMC".

\section{Diacronie: Le terme "Digital History" est utilisé pour indiquer de façon générique l'utilisation des outils informatiques en histoire. Quels sont, à votre avis, ses principales composantes?}

Claire Lemercier: Il me semble que le terme est principalement utilisé aujourd'hui par des collègues qui réalisent une édition électronique de sources - par capillarité à partir des "digital humanities" 8 , sans doute. D’une manière qui pourrait sembler paradoxale, ainsi, la "digital history" aurait surtout sa place en histoire ancienne et médiévale et/ou dans les domaines de l'histoire les plus attachés à la notion de grands textes (histoire de la littérature, histoire des sciences, etc.) ou encore qui travaillent avec l'image (histoire des images, mais aussi cartographie historique par exemple), plutôt qu'en histoire contemporaine ou chez les historiens se revendiquant des sciences sociales. On n'entend pratiquement jamais l'expression "digital social science”!

Pourtant, dans ma pratique d'historienne du contemporain proche de la sociologie, j'utilise en permanence des outils informatiques : comme nous tous pour accéder à la bibliographie et aux sources, pour réaliser une veille scientifique, pour communiquer avec les collègues, pour écrire des textes, notamment à plusieurs ; et, un peu plus que d'autres, pour une activité d'éditrice, ainsi que pour constituer des bases de données à partir de sources et pour les soumettre à différents traitements quantitatifs ou, plus largement, formels. Aucun aspect de mon activité professionnelle ne se fait sans informatique!

Mais cette omniprésence conduit, il me semble, à une dilution de la notion de "digital history”, dont on peut du coup se demander si elle est bien utile. Si elle doit désigner

\footnotetext{
5 Revue «Histoire \& Mesure» URL : < http://histoiremesure.revues.org/ > [consulté le 3 mai 2012].

${ }^{6}$ Lire en ligne sur TEL, URL : < http://tel.archives-ouvertes.fr/tel-00412011 > [consulté le 3 mai 2012].

7 Quanti IHMC, URL : < http://www.quanti.ihmc.ens.fr/ > [consulté le 3 mai 2012].

8 Sur cette notion, voir INTD-CNAM, «Digital humanities», in Digital humanities international. Veille sur les Humanités Numériques et champs associés, sans date,

URL : < http://dhi.intd.cnam.fr/digital-humanities/ > [consulté le 3 mai 2012].
} 
dans leur ensemble les pratiques des historiens un peu geeks, ceux que leurs collègues appellent quand ils rencontrent un problème "informatique", elle ne me semble pas très intéressante: elle mélange des savoir-faire très différents et contribue à maintenir l’idée d'un ghetto, d'une forme d'histoire qui ne serait accessible et féconde que pour quelques-uns.

Il me semble donc qu'il est urgent de lui donner un contenu plus précis. Je ne suis pas plus légitime qu'une autre pour en proposer un, d'autant que je ne suis pas très intégrée dans les discussions internes aux "digital humanities". Mais il me semble qu'un aspect fondamental est la question de la structuration de l'information historique. Il n'a rien de propre, en soi, à l'informatique : la question se posait auparavant. Mais la quantité d'information accessible et les outils disponibles pour la traiter rendent cette question à la fois plus urgente et plus traitable. Pour moi, le plus important en matière de formation des apprentis historiens à la "digital history" est donc de leur apprendre à constituer des bases de données bibliographiques avec des outils de type Zotero ${ }^{9}$ (trouver l'information et structurer les métadonnées pour qu'elles soient utiles à leur recherche) et des bases de données en général à partir de sources (structurer de l'information en lignes et en colonnes sans perdre les acquis de la discipline historique, c'est-à-dire en gardant le lien avec les sources et un équilibre entre le langage de la source et les questions de l'historienne). Ce sont des questions qui se posaient déjà à l'époque des fiches cartonnées! Mais si le label "digital history" peut les rendre plus explicites et plus centrales dans la discipline, tant mieux.

\section{D.: Depuis une bonne dizaine d'année, l'utilisation des technologies numériques en histoire a connu un développement très rapide. De votre point de vue, quelles sont les principales transformations du métier d'historien qui en ont découlé?}

C.L.: Tout a changé! Même si la bonne manière de réagir à ces changements est sans doute de garder les bases de notre métier (la critique des sources notamment) comme boussole : tout a changé dans les manières de faire, sans que cela oblige à changer de principes. Si l'on compare avec la situation d'il y a vingt ans, c'est encore plus frappant,

\footnotetext{
9 Zotero, URL : < http://www.zotero.org/ > [consulté le 3 mai 2012]. Sur Zotero on peut aussi voir : HEIMBURGER, Franziska, RUIZ, Émilien, «Has the Historian's craft gone digital? Some observations from France», in Diacronie. Studi di Storia Contemporanea: Digital History: la storia nell'era dell'accesso,

URL:< http://www.studistorici.com/2012/o6/29/clavert_numero_10/ > [consulté le 29 juin 2012].
} 
même si d'autres évolutions ont eu lieu depuis dix ans (plutôt, il me semble, sous forme d'une extension des évolutions pionnières). D’abord, même si c'est sans doute ce dont on discute le moins, il y a la multiplication des e-mails. Ils finissent par gêner le travail de recherche classique, le diptyque constitué par le travail dans les sources et l'écriture, tant on passe de temps à y répondre, avec une pression croissante pour y répondre vite. Mais ils ont incroyablement accru la communication scientifique à l'échelle mondiale (surtout si on y inclut les listes de diffusion/discussion) et l'ont rendue plus facile : même si toutes les barrières hiérarchiques et linguistiques sont loin d'avoir disparu, on a toutes les chances de localiser quelqu'un qui travaille sur le même sujet mais très loin de nous et d'arriver à interagir avec lui.

La numérisation de revues et maintenant, petit à petit, de livres, a le même effet : cela nous insère dans un espace de dialogue, par la citation ou la discussion directe, bien plus vaste. C'est une situation pour laquelle nous ne sommes pas encore vraiment armés : il y a un vertige de la bibliographie lisible et des contacts possibles. Où s'arrêter ? Comment savoir si un membre de jury ne va pas nous coincer sur un élément qui aurait été pertinent mais que nous n'avons pas localisé ou pas lu ? Comment être sûr, même, que ce qu'on est en train d'étudier ne l'a pas déjà été ? Auparavant, on n'en était pas sûr, mais on savait qu'il fallait vivre avec. Aujourd'hui, il faut décider du moment où on arrête de vérifier.

L'autre grand changement concerne l'accès aux sources et la manière de les lire. En histoire contemporaine, et notamment pour le XIX ${ }^{\mathrm{e}}$ siècle, sur lequel je travaille la plupart du temps, des monceaux de sources sont devenus accessibles, souvent avec une possibilité de recherche en texte intégral, mais en ordre dispersé plutôt que sous forme de corpus cohérents, et souvent avec une accessibilité limitée à certaines institutions (je pense aux extraordinaires bases de l'entreprise Gale ${ }^{10}$, auxquelles presque aucune université en France n'est abonnée).

Plus largement, la révolution dans le rapport aux sources vient de la photographie numérique, à la pratique de laquelle, là encore, les historiens restent très mal formés : Comment indexer efficacement les photos ? Faut-il encore recopier intégralement des portions du texte des sources, et à quel stade de la recherche ? Les réponses viennent de jeunes collègues qui n'ont pas honte d'exposer de telles questions pratiques (voir notamment la Boîte à outils des historiens ${ }^{11}$ ), mais elles sont encore trop peu discutées collectivement.

${ }^{10}$ Gale. Cengage Learning, URL: < http://www.gale.cengage.com/ > [consulté le 3 mai 2012].

${ }^{11}$ La boîte à outils des historiens, URL: < http://www.boiteaoutils.info > [consulté le 3 mai 2012]. 
Tout cela a rendu accessibles beaucoup plus de sources qu'auparavant, notamment en réduisant le temps à passer dans leur lieu de conservation, parfois à zéro : cela devrait inciter à réfléchir sur la nécessité de se poser malgré tout des questions sur l'origine des fonds et la matérialité des sources, questions qui sont plus évidentes lorsqu'on les a physiquement entre les mains. Mais le problème crucial, comme pour la bibliographie, est celui de l'arbitrage entre temps de collecte d'information et temps de lecture et de traitement : on peut assez vite remplir son ordinateur d'articles, de livres anciens numérisés et de photographies. Mais quand et comment les lire, comment circuler entre eux quand la masse ne permet plus de tout garder en tête - ce qui était possible lorsque, nécessité faisant loi, nous travaillions sur de plus petits corpus?

\section{D.: Cette facilitation des échanges scientifiques ne pose-t-elle pas aussi la question du développement des recherches collectives en sciences sociales? D'autant que pour la collecte et la transcription de sources, l'élaboration de bibliographies, voire l'écriture elle-même, des outils de travail collaboratif permettent désormais de contourner la plupart des obstacles “techniques” à ce type de démarches.}

C.L.: Il est vrai que pour moi qui écris beaucoup à quatre mains ou plus, les outils les plus basiques de révision de documents ou de partage en ligne sont cruciaux. Même s'ils laissent encore beaucoup à désirer (on finit toujours par perdre des éléments en passant d'un ordinateur à un autre configuré très différemment), ils ont eu un effet très positif, principalement d'ordre psychologique, je crois, en rendant plus transparente l'intervention de chacun et plus faciles les retours en arrière. On se froisse moins des révisions des autres lorsque leur intervention peut être précisément retracée.

J'ai moins expérimenté pour l'instant les bibliographies partagées ou dépôts partagés de sources. Je m'y mets toutefois, dans le cadre de nouvelles recherches collectives : je suis en pleine confrontation aux embûches pratiques (s'accorder sur des manières de nommer les fichiers, utiliser une Dropbox avec une connexion rurale flageolante...), mais évidemment, cela rend possible des choses qui ne l'étaient pas auparavant, en particulier un partage assez commode du travail de dépouillement, si l'on se donne mutuellement accès à la fois aux notes mais aussi, en cas de doutes ou de lectures différentes, au matériau primaire. Pour l'heure, j'ai tout de même le sentiment que ce type d'outils nécessite non seulement un même niveau de familiarité avec le numérique, mais aussi une harmonisation des équipements informatiques au sein des équipes, ce qui peut être un peu lourd. 
Cela dit, ces outils peuvent faciliter les collaborations à distance, même s'ils ne dispensent pas d'organiser des rencontres physiques, en particulier pour apprendre à se connaitre dans des interactions plus informelles, ce qui reste crucial. Mais ce ne sont de toute façon pas les outils qui vont faciliter le processus de recherche collective en luimême. En effet, celui-ci se heurte toujours, dans nos disciplines, à des obstacles institutionnels: même si les institutions poussent aux projets collaboratifs nous restons jugés, lorsqu'il s'agit de carrières, principalement sur la base de travaux individuels, comme la thèse ou l'HDR; on accorde même souvent moins de considération aux publications cosignées - sans parler des productions utiles à tous, mais difficiles à faire entrer dans les cases des formulaires d'évaluation, comme les bases de données ou les carnets de veille. Et même l'injonction aux projets collaboratifs ignore trop la difficulté qu'il y a à s'accorder sur les objectifs d'une recherche et sur une division du travail : tout cela ne va pas de soi, surtout dans un milieu où on peut rarement faire appel à l'autorité hiérarchique. Les outils numériques peuvent aider à expliciter les problèmes potentiels assez en amont : qui aura accès à quoi, en échange de quoi ? Ils peuvent donc éventuellement faciliter une négociation, mais ils n'en garantissent pas à eux seuls l'issue...

D.: Le projet Zotero Commons ${ }^{12}$ propose de mettre en commun les numérisations d'archives auxquelles nous procédons lorsque nous prenons des photos aux archives, par exemple. L'ensemble est stocké sur Internet Archive'13, qui procède automatiquement à une reconnaissance optique des documents soumis (OCR). Un tel projet, s'il semble prometteur, nous rappelle que certains outils de travail collaboratif sont parfois en avance sur le droit. Cela ajoute une difficulté à celles que vous évoquez. Qu'en pensez-vous?

C.L.: J'utilise beaucoup par ailleurs Internet Archive, que ce soit pour accéder à l'archivage du web, pour mettre en ligne des enregistrements de séminaires ou pour consulter des imprimés numérisés - et là aussi, cela donne parfois accès à des imprimés auxquels on n'a normalement pas droit par le biais de Google Books par exemple. Il me semble qu'ici, le droit suivra les pratiques : de toute façon, nombre de photographies de sources concernent des documents libres de droits, mais souvent protégés en pratique

${ }_{12}$ Zotero Commons, URL: < http://www.zotero.org/support/commons > [consulté le 3 mai 2012].

${ }^{13}$ Internet Archive, URL: < http://archive.org/ > [consulté le 3 mai 2012]. 
sans grandes raisons de fond par des bibliothèques, par exemple. Tant mieux s'ils sont effectivement rendus accessibles. Et même lorsque les documents sont en théorie protégés, on peut s'interroger sur ceux qui sont épuisés, par exemple. Il me semble qu'il faut développer la culture juridique des utilisateurs et des éditeurs, par exemple à propos des licences Creative Commons ${ }^{14}$ qui permettent de prévoir seulement une utilisation à but non lucratif, et qu'une convergence finira par être trouvée : on ne parle pas ici d'enjeux financiers comme ceux de l'industrie du disque.

En revanche, ce qui me préoccupe chez Internet Archive, comme d'ailleurs Google Books, c'est l’indigence des métadonnées, très mal ou pas du tout renseignées, qui rendent impossible la recherche par date de publication, notamment (sans parler des éditeurs) et aléatoire celle par titre ou par auteur. On voit là ce qu'on perd quand on n'a pas de bibliothécaire (alors même que nous avions longtemps critiqué les défauts de Gallica...)! Tout projet collaboratif se heurte à cet écueil: il est difficile de bien documenter les sources numérisées, et pourtant c'est absolument essentiel si on veut qu'elles servent à d'autres. Cet aspect est donc pour moi essentiel dans tout partage de source - comme la possibilité de correction collaborative de l'OCR, certes un travail de Sisyphe, mais des vaillants retraités ou autres s'y sont bien mis ici et là... et là encore c'est in fine une condition nécessaire si on veut vraiment profiter des moteurs de recherche.

\section{D.: Le développement du libre accès à l'information scientifique a aussi} grandement facilité la consultation de travaux universitaires - à travers les "archives ouvertes" notamment -, qu'ils soient publiés (articles, ouvrages...) ou non (mémoires, thèses, mais aussi communications à des colloques), tout en suscitant certaines réserves, voire de la méfiance, de la part de chercheurs ou d'éditeurs scientifiques. Vous êtes vous-même impliquée dans diverses activités d'édition scientifique, et vous avez déposé de nombreux travaux sur HAL-SHS. Comment vous positionnezvous par rapport à ce mouvement?

C.L.: Je n'ai pas changé de position sur ce point depuis le début : le libre accès me semble devoir être la règle. C'est en partie une question de principe : en France en tout cas, les producteurs de travaux scientifiques en SHS sont dans leur immense majorité fonctionnaires ou bénévoles, je ne vois donc pas de raison pour que leurs productions

\footnotetext{
14 Creative Commons France, URL : < http://creativecommons.fr/ > [consulté le 3 mai 2012].
} 
ne bénéficient pas à tous ${ }^{15}$. Ces travaux n'ont en outre pas, en général, de grandes perspectives lucratives. Et les acteurs privés de l'édition qui s'opposent au libre accès n'offrent malheureusement, souvent, qu'un enrichissement minime à ces productions : combien d'éditeurs qui demandent des manuscrits déjà corrigés, voire déjà mis en page, et se contentent d'envoyer le tout à divers sous-traitants (imprimeur, routeur), sans même essayer de mettre le livre ou la revue en valeur ${ }^{16}$ ? On n'est alors pas si loin de l'impression à la demande ou des archives ouvertes, même si le papier garde un prestige propre pour beaucoup de collègues... (Heureusement, ce n'est pas ce que fait La Découverte, avec qui je travaille: la vente à la découpe des Repères proposée par Cairn est au contraire bien moins ergonomique et moins avantageuse que leur lecture sur papier !).

De plus, le libre accès me paraît avoir une raison pratique déterminante : il existe de fait pour les personnes bien insérées dans la communauté. Je ne paye que très rarement pour accéder à un livre ou un article, car mes institutions d'appartenance ont les bons abonnements ou les bonnes bibliothèques, je reçois des livres pour compte rendu ou par des amis, et je peux en dernier recours demander un chapitre ou article par e-mail à son auteur.e... Si, dans le même temps, un étudiant en M1, un universitaire d'un pays du Sud, ou simplement d'une petite université française, ou un citoyen intéressé doit payer, il me semble qu'il y a maldonne.

C'est pour cela que je dépose le plus possible mes publications sur HAL-SHS, éventuellement avec un petit délai si je pense que l'éditeur pourrait être froissé. Et je commence à constater (par des citations ou des contacts interpersonnels) qu'elles sont souvent lues là, sans doute plus que sur papier pour les contributions à des livres collectifs obscurs... Est-ce un problème ? Il me semble au contraire que cela ne fait que valoriser ces livres collectifs qui font parfois l'objet d'un important travail de leurs directeurs mais restent toujours mal aimés des maisons d'édition. Toutefois, la mise à disposition d'articles de revues ou de livres collectifs sur le web a aussi pour conséquence de casser l'unité des volumes, parfois au détriment de la réelle ligne éditoriale qui y était promue. C'est une des choses qui me fait préférer, comme utilisatrice, Revues.org, qui identifie au moins visuellement les revues, à Cairn, qui les

15 C'est d'ailleurs la position officielle de nombreuses institutions, notamment celle des institutions européennes depuis plusieurs années. Voir les textes de référence alignés par l'Institut de l'Information Scientifique et Technique du CNRS (Inist-Cnrs) sur le site Libre accès à l'information scientifique et techniques. Actualités, problématiques et perspectives,

URL: < http://openaccess.inist.fr/ > [consulté le 3 mai 2012].

${ }^{16}$ Pour un exemple récent de ces dérives qui touchent même des éditeurs de référence, voir MONITI, Luca, «De la mondialisation appliquée à l'édition», in Le Tigre, 6, 2011, pp. 34-37. En ligne depuis le 11 octobre 2011. URL : < http://www.le-tigre.net/De-la-mondialisationappliquee-a-l.html > [consulté le 3 mai 2012]. 
homogénéise. Mais cela peut se régler par une éducation des lecteurs, étudiants notamment : il faut leur apprendre à remonter d'un texte à son contexte de publication pour mieux le comprendre.

De la même manière, une partie de la méfiance envers le libre accès pourrait être éradiquée par l'éducation : pour beaucoup de collègues, pas toujours de mauvaise foi, un article en ligne vaut toujours moins qu'un article sur papier, parce qu'ils n'imaginent pas qu'un article nativement numérique ait pu être soumis à un comité de rédaction tout aussi exigeant. J'ai du mal à penser que cette position puisse résister face à la réalité - y compris celle des classements officiels de revues qui, dans plusieurs disciplines, commencent à intégrer des revues purement numériques - mais elle persiste déjà depuis 10 ans...

Sans doute y a-t-il eu un problème de communication lié à la célébration conjointe de la mise en ligne de matériaux sélectionnés scientifiquement, d'une part, et de l'ouverture d'“archives ouvertes" pour des objets non publiés, d'autre part. Je trouve très important de pouvoir mettre en ligne et lire de la "littérature grise" : des mémoires ou thèses, qui ont en réalité fait l'objet d'une validation scientifique, mais aussi des écrits plus préliminaires. Les économistes le font bien plus que nous et j'utilise largement leurs "working papers”; c'est très intéressant de mettre ainsi en ligne un objet imparfait et d'obtenir des commentaires à son sujet. Mais il faut que le caractère préliminaire et non validé de l'objet soit bien clair pour tous.

\section{D.: Vous avez soutenu votre thèse de doctorat en histoire en 2001 et vous venez de soutenir votre habilitation à diriger des recherches. Dans ce laps de temps, qu'est-ce que le passage à“l'ère numérique” a le plus changé dans vos pratiques d'historienne?}

C.L.: Pour moi, le principal changement concerne la question de gestion de la masse que j'ai déjà évoquée. J'ai encore chez moi une étagère pleine des cartons concernant ma thèse : photocopies d'éléments de bibliographie et de sources, notes manuscrites et imprimées sur d'autres sources. Ce n'est pas une très grande étagère et tous les matériaux utilisés sont là. En revanche, pour mon HDR, les choses sont beaucoup plus dispersées ; certains éléments n'existent que sur papier, d'autres seulement sous forme de fichiers, d'autres sous les deux formes, et surtout tout cela est bien plus volumineux - et c'est bien plus une conséquence des nouvelles formes d'accès à l'information que de l'ambition plus large du projet. Quand je me suis mise à rédiger, j'ai néanmoins, pour les premiers chapitres, procédé comme pour la thèse : je relisais l'ensemble des 
éléments de sources et de bibliographie que j'avais rangés dans des dossiers physiques ou virtuels correspondant à ces chapitres (ou je survolais mes annotations en leur sein), avant ou pendant la rédaction. Mais je n'ai pas pu le faire pour tous les chapitres : cela aurait été trop long. Paradoxalement, alors que le matériau était souvent dans l'ordinateur même, j'ai plus rédigé “de tête”. Et les corpus n'étaient jamais vraiment clos, car la tentation d'aller chercher un élément supplémentaire en ligne était trop forte pour y résister (alors que, s'il avait fallu retourner en archives ou en bibliothèque, souvent, je ne l'aurais pas fait).

D'où un plus grand sentiment de doute et de frustration, dont on ne peut sortir qu'en se demandant en permanence si les éléments manquants sont vraiment centraux pour la vision personnelle que l'on veut donner du sujet. C'est épuisant pour les nerfs, mais c'est sans doute plutôt bon, à la fin, pour la recherche en histoire. Nous avons été élevés dans la religion de l'exhaustivité : voir toutes les sources et toute la bibliographie sur un sujet - l’implicite, énorme, étant "toutes celles qui étaient accessibles là où nous faisions notre thèse". Il n'est plus possible de raisonner ainsi aujourd'hui, même si malheureusement bien des membres de jurys (pas ceux de mon HDR!) le font encore. Il faut choisir délibérément, en fonction d'une problématique. Cela peut être un bien si cela nous éloigne d'une conception trop antiquaire de l'histoire!

\section{D.: Au quotidien, dans le cadre de vos propres travaux, quels sont les outils informatiques spécifiques ou les ressources numériques particulières que vous mobilisez qui ont le plus affecté vos pratiques?}

C.L.: J'utilise probablement plus d'outils de veille que la moyenne des historiens, sans doute pas très "proprement"d'ailleurs sur un plan technique, puisque je les fais en général converger vers ma boîte à courrier électronique (plutôt que d'utiliser un lecteur de flux RSS, par exemple). Et je peste régulièrement sur le temps que cela me prend, car je ne peux pas m'empêcher de regarder ce que ces outils me ramènent, parfois aux dépens d'une tâche d'écriture urgente. Mais avec le recul, je crois que me tenir au courant de ce qui se passe dans mes domaines de spécialité étroite, mais aussi un peu plus largement est crucial pour l'évolution de mes recherches comme pour la qualité de mon enseignement. Ainsi, j'épluche les lettres d'Openedition ${ }^{17}$ et Liens-Socio ${ }^{18}$, les sommaires de revues proposés par certains éditeurs ou portails, les digests de diverses

${ }_{17}$ OpenEdition. Portail de ressources électroniques en sciences humaines et sociales, URL: < http://www.openedition.org/ > [consulté le 3 mai 2012].

${ }^{18}$ Liens socio. Portail francophone généraliste d’informations en sciences sociales, URL : < http://www.liens-socio.org/ > [consulté le 3 mai 2012]. 
listes de diffusion ou discussion (histoire_eco ${ }^{19}$, H-France ${ }^{20}$, H-Reviews ${ }^{21}, \mathrm{H}-$ Business $^{22}$, eh.net ${ }^{23}$, Criminocorpus ${ }^{24} .$. ), j'ai quelques alertes Google 25 et je suis différents carnets de recherche, notamment ceux qui traitent de méthodes quantitatives ou du métier en général (sur Hypothèses ${ }^{26}$, Quanti ${ }^{27}$, Devenir historien ${ }^{28}$, Polit'bistro ${ }^{29}$, Aspects concrets de la thèse ${ }^{30}$, ailleurs notamment le blog de Baptiste Coulmont ${ }^{31}$ ). C'est ma manière de faire des pauses sur mon ordinateur. Cela prend un temps croissant, qui mord à la fois sur mes loisirs et sur d'autres activités professionnelles ; je dois d'ailleurs arrêter en période de travail vraiment intense (écriture de l'HDR, préparation d'un nouveau cours). Mais c'est indispensable le reste du temps - et cela ne fait que prendre le relais de mes pratiques d'avant 2000 : feuilleter les derniers numéros de toutes les revues en bibliothèque, regarder les affiches de colloques...

Par ailleurs, j'utilise beaucoup d'outils de traitement quantitatif ou formel, très importants pour ma recherche, puisque je vise en permanence à éclairer le quantitatif par le qualitatif, et vice versa. Par rapport aux outils que j'avais découvert, parfois sous DOS, vers 1997, le changement en la matière a été très progressif : ce n'est pas un changement de nature, même si les logiciels sont à la fois plus puissants et plus souples, et plus souvent libres et gratuits. Mais la logique générale de leur fonctionnement reste la même : il faut surtout apprendre à structurer l'information en amont, "bricoler" pour transférer des données d'un environnement à un autre, et, à un moment, sauter le pas de la programmation, fût-ce de manière très simple et progressive. Ce qui est le plus nouveau, pour moi, c'est que la souplesse croissante des logiciels d'analyse formelle, en particulier en termes de formats de données acceptés (en gros, il n'est plus nécessaire d'appliquer des codages trop violents), permet leur application à des recherches sur les thèmes les plus variés. Comme la gratuité les rend aussi, souvent, plus accessibles, il est devenu d'autant plus important pour moi de dispenser des formations dans ce

${ }_{19}$ Actualité de la recherche et de l'enseignement en histoire économique [liste de diffusion], URL : < https://groupes.renater.fr/sympa/info/histoire_eco/ > [consulté le 3 mai 2012].

${ }^{20} \mathrm{H}$-France, URL: < http://www.h-france.net/ > [consulté le 3 mai 2012].

${ }^{21} \mathrm{H}$-Net Reviews in the Humanities and Social Sciences,

URL : < http://www.h-net.org/reviews/home.php > [consulté le 3 mai 2012].

${ }^{22}$ H-Business, URL: < http://www.h-net.org/ business/ > [consulté le 3 mai 2012].

${ }^{23}$ EH.net, URL : < https://eh.net/ > [consulté le 3 mai 2012].

${ }_{24}$ Criminocorpus. Portail sur l'histoire de la Justice, des crimes et des peines,

URL : < http://www.criminocorpus.cnrs.fr/ > [consulté le 3 mai 2012].

${ }_{25}$ Google Alertes, URL : < http://www.google.com/alerts?hl=fr> [consulté le 3 mai 2012].

${ }^{26}$ Hypotheses.org. Academic blogs, URL : < http://hypotheses.org/ > [consulté le 3 mai 2012].

${ }^{27}$ Quanti/ sciences sociales, URL : < http://quanti.hypotheses.org/ > [consulté le 3 mai 2012].

${ }_{28}^{28}$ Devenir historien-ne, URL: < http://devhist.hypotheses.org/ > [consulté le 3 mai 2012].

${ }^{29}$ Polit'bistro : des politiques, du café, URL: < http://politbistro.hypotheses.org/> [consulté le 3 mai 2012].

${ }^{30}$ Les aspects concrets de la thèse, URL: < http://act.hypotheses.org/> [consulté le 3 mai 2012].

${ }^{31}$ Baptiste Coulmont, URL : < http://coulmont.com/blog/ > [consulté le 3 mai 2012]. 
domaine, afin que ces outils ne restent pas réservés à un ghetto de quantitativistes qui ne feraient de l'histoire que de cette façon, mais qu'ils deviennent un auxiliaire parmi d'autres de la recherche, pour tout le monde.

D.: Vous évoquez la lecture de carnets de recherche et vous tenez vousmême, avec Claire Zalc, un site web "Quanti IHMC". Depuis quelques années, le "blogging scientifique" est une pratique qui s'est développée en France. Toutefois, presque paradoxalement (compte tenu du rôle qu'ont pu jouer certains d'entre eux dans la fondation et le développement de plateformes telles qu'Hypothèses ou Culture Visuelle ${ }^{32}$ ), elle reste peu adoptée par les historiens. Que pensez-vous de cette nouvelle forme d'écriture scientifique? Comment expliquer le faible engouement des historiens pour ce nouvel outil?

C.L.: Je ne sais pas si l'engouement des historiens est vraiment faible, il faudrait l'objectiver... Il me semble là encore qu'il est plus fort chez ceux qui s'identifient aux "humanities", à l'édition de corpus par exemple. En tout cas, il est vrai qu'il n'est pas très fort chez les collègues que je côtoie en général. Et moi-même, en réalité, je ne blogue pas. Notre site Quanti a été pensé comme site (dérivé de mon site personnel, adapté comme compagnon d'un livre) avant la vogue des blogs scientifiques, et son contenu s'en ressent : rares y sont les vrais billets, rédigés comme tels, avec l'alliance d'un contenu sérieux et d'un ton plus informel que celui d'un article scientifique, d'un cours ou d'une notice de logiciel. J'ai écrit quelques billets de ce type pour un autre blog de discussion sur notre métier33, mais ce n'était pas pour parler de mes recherches, et j'ai dû arrêter pendant la rédaction de mon HDR : mon cerveau n'arrivait pas à mener de front les deux activités. En réalité, je n'arrive pas à trouver une place dans mon emploi du temps, et plus fondamentalement dans mes envies, dans l'emploi de mon énergie intellectuelle, pour l'écriture d'un carnet de recherche au sens plein.

Je constate pourtant que d'autres y arrivent très bien, sans savoir si c'est vraiment une question de discipline. C'est possible pour les économistes, dont les carrières s'articulent autour d'articles, en assez petit nombre mais très peaufinés, écrits en anglais et très formalisés : peut-être ont-ils d'autant plus besoin d'un espace pour écrire

$3^{2}$ Culture visuelle. Média social d'enseignement et de recherche, URL: < http://culturevisuelle.org/ > [consulté le 3 mai 2012].

33 Évaluation de la recherche en SHS. URL : <http://evaluation.hypotheses.org> [consulté le 3 mai 2012]. 
des choses plus vulgarisées et en français ${ }^{34}$. D'ailleurs il me semble que les économistes qui bloguent écrivent souvent aussi des Repères, par exemple ; ce n'est sans doute pas par hasard. Peut-être que les historiens ont déjà beaucoup d'autres lieux pour faire ce genre de choses, par exemple en allant parler sur France Culture (ce que j'aime bien faire, mais qui ne touche évidemment pas tout à fait le même public) ou plus généralement en étant sollicités par d'autres médias?

Dans mon cas toutefois, je crois que l'incapacité à bloguer est liée à ma manière d'écrire sur mes recherches : je ne le fais que contrainte et forcée et j'ai beaucoup de mal à écrire des premiers jets, parce que la réflexion vient en écrivant et qu'elle vient en fonction de la forme précise à adopter, article ou livre, en français ou anglais, etc. (j'ai même du mal à faire des plans détaillés). Il n’y a pas de place là-dedans pour une préformalisation sous forme de billet, même si je trouve cela dommage et n'ai pas tout à fait renoncé à essayer. En revanche, je ne peux qu'inciter les jeunes chercheurs à faire leurs gammes dans des carnets de recherche, que ce soit pour apprendre à écrire des recensions ${ }^{35}$ ou pour tester sous une forme résumée les arguments d'un mémoire ou d'une partie de mémoire de recherche.

\section{D.: Quelles sont, selon vous, les prochains défis que la "digital history" devra relever? Quels sont les domaines qui vous paraissent les plus fructueux pour des développements ultérieurs?}

C.L.: Pour moi, le défi est celui de la normalisation : permettre l'appropriation par tous des outils de la "digital history", pour que ce terme, à la limite, devienne inutile parce qu'elle se confondrait avec l'histoire tout court. Il y a toujours besoin de développements informatiques à proprement parler, par exemple de bons logiciels libres de lexicométrie (un domaine encore très propriétaire, avec tout ce que cela implique en termes de coûts et de boîtes noires) ${ }^{36}$. Mais, sans doute aussi parce que je ne suis vraiment experte d'aucun outil tout en en utilisant beaucoup, je pense que

\footnotetext{
34 J'apprécie en particulier ce blog de doctorants : Ma femme est une économiste (et moi aussi), URL : < http://www.mafeco.fr/ > [consulté le 3 mai 2012] mais, comme ses liens en témoignent, des chercheurs en poste bloguent aussi.

35 Voir à ce sujet CHEVALIER, Clara, RUIZ, Émilien, «Comment (et pourquoi) écrire un compte rendu de lecture?», in Devenir historien-ne, 29 septembre 2011, URL : <http://devhist.hypotheses.org/492> [consulté le 3 mai 2012].

36 Des espoirs sont toutefois permis : voir Textométrie. Fédération des recherches et développements en textométrie autour de la création d'une plateforme logicielle ouverte, URL: < http://textometrie.ens-lyon.fr/ > [consulté le 3 mai 2012] et plus généralement CINQUIN, Sophie, «Utiliser la lexicométrie en histoire (2) : aspects concrets», in Devenir historien-ne, 16 mars 2012, URL : <http://devhist.hypotheses.org/1068> [consulté le 3 mai 2012].
} 
l'urgence est moins de développer de nouveaux logiciels que de mettre l'accent sur la documentation et la formation.

Plus exactement, les historiens geeks ont besoin de mieux parler à deux publics. D’un côté, les informaticiens : il y en a beaucoup qui ne demandent qu'à travailler avec nous, mais sans toujours se rendre compte des spécificités de nos matériaux et de nos métiers ; il faut donc mieux apprendre à se parler (et fort heureusement beaucoup de dialogues de ce type sont déjà en cours) pour travailler ensemble, et non pas pour que chacun s'approprie, mal, le métier de l'autre. Et je pense autant aux spécialistes des interfaces, capables de proposer des outils d'analyse ou de visualisation parlants à l'historien lambda ${ }^{37}$, qu'aux spécialistes du data mining ou du calcul...

De l'autre côté, et c'est plutôt sur cet aspect que je m'investis, il faut que les geeks parlent avec les jeunes chercheurs en formation - et si possible avec les chercheurs en poste de tout âge... Il faut inventer une manière de leur présenter des outils non pas en tant que gadgets et en leur apprenant où cliquer, mais bien en lien avec les bases de notre métier, de montrer en quoi ces outils sont nécessaires pour conserver ces bases, et non pas pour leur ajouter un vernis moderne. Pour moi, le véritable intérêt des outils informatiques, c'est finalement qu'ils nous amènent à parler des méthodes, des pratiques propres à notre métier, comme la lecture et la prise de note sur une source, dont il était trop souvent admis, lorsque j'ai débuté, qu'on ne pouvait l'apprendre que sur le tas, en faisant mais sans jamais en discuter collectivement. Tout cela nous rappelle que ce que nous faisons est un véritable travail, qui ne va pas de soi, et dont on peut améliorer les outils, sans craindre pour autant qu'ils nous remplacent.

37 Par exemple l'équipe de Jean-Daniel Fekete, un grand artisan du dialogue avec les sciences humaines et sociales. URL: < http://www.lri.fr/ fekete/ > [consulté le 3 mai 2012]. 


\section{* Les auteurs}

Elisa Grandi est doctorante en histoire contemporaine et histoire de l'Amérique Latine à l'Université Paris Diderot et membre du laboratoire SEDET. Sa thèse Réseaux Sociaux et changement institutionnel. Experts internationaux, médiation politique et reformes économiques en Colombie (1949-1966), sous la direction de Zacarias MOUTOUKIAS, porte sur les réseaux reliant les experts de la Banque Mondiale et le personnel local en Colombie lors des premières missions de la Banque dans le pays. Après avoir été Visiting Student à la Duke University et Visiting Scholar à la New School of Social Research, elle est actuellement boursière du Gouvernement français Eiffel-Excellence à l'Université Paris Diderot. Parmi ses publications: «David Lilienthal, la Banca Mondiale e lo sviluppo una rete transnazionale di economic advising (1950-1957)», Diacronie. Studi di Storia Contemporanea: Storia transnazionale e prospettive transnazionali nell'analisi storica, N. 6, 2|2011.

URL: < http://studistorici.com/2010/12/o7/elisa_grandi/ >

Emilien Ruiz est doctorant en histoire contemporaine à l'Ecole des Hautes Etudes en Sciences Sociales (EHESS). Sa thèse Trop de fonctionnaires? La question des effectifs de l'État dans la France du XXe siècle porte sur divers aspects de l'évolution du nombre des fonctionnaires de l'État français des années 1880 aux années 1980. Il a enseigné la méthodologie de la recherche en histoire, l'histoire contemporaine et l'informatique pour les sciences historiques à l'EHESS et à l'Université Paris Diderot. Depuis janvier 2012, il est assistant de Paul-André Rosental à Sciences po. Il anime deux blogs scientifiques: La Boite à Outils des Historiens (avec Franziska Heimburger), sur les outils informatique pour l'histoire; Devenir historien-ne, sur la méthodologie de la recherche en histoire. Parmi ses publications: «Compter: l'invention de la statistique des fonctionnaires en France (années 1890-1930)», in BEZES, Philippe, JOINLAMBERT, Odile (dir.), «Comment se font les administrations», Sociologie du Travail, 52, 2/2010, pp. 212-233.

URL: < http://studistorici.com/2010/12/o7/emilien_ruiz/ >

\section{Per citare questo articolo:}

GRANDI, Elisa, RUIZ, Émilien, "Ce que le numérique fait à l'historien.ne. Entretien avec Claire Lemercier», Diacronie. Studi di Storia Contemporanea: Digital History: la storia nell'era dell'accesso, 29/6/2012, URL:< http://www.studistorici.com/2012/06/29/grandi_numero_10/ > 
Diacronie Studi di Storia Contemporanea $\sqrt{3}$ www.diacronie.it

Risorsa digitale indipendente a carattere storiografico. Uscita trimestrale. redazione.diacronie@hotmail.it

Comitato di redazione: Marco Abram - Giampaolo Amodei - Jacopo Bassi - Luca Bufarale - Alessandro Cattunar - Alice De Rensis Barbara Galimberti - Deborah Paci - Fausto Pietrancosta - Matteo Tomasoni - Luca Zuccolo

CC () $\odot$ Diritti: gli articoli di Diacronie. Studi di Storia Contemporanea sono pubblicati sotto licenza Creative Commons 2.5

(C) Possono essere riprodotti a patto di non modificarne i contenuti e di non usarli per fini commerciali. La citazione di

BY NC ND estratti è comunque sempre autorizzata, nei limiti previsti dalla legge. 